\title{
Independent individual addressing of multiple neutral atom qubits with a micromirror-based beam steering system
}

\author{
C. Knoernschild, ${ }^{1}$ X. L. Zhang, ${ }^{2}$ L. Isenhower, ${ }^{2}$ A. T. Gill, ${ }^{2}$ F. P. Lu, ${ }^{3}$ M. Saffman, ${ }^{2}$ and \\ J. $\mathrm{Kim}^{1,3, a)}$ \\ ${ }^{1}$ Department of Electrical and Computer Engineering, Fitzpatrick Institute for Photonics, Duke University, \\ Durham, North Carolina 27708, USA \\ ${ }^{2}$ Department of Physics, University of Wisconsin, 1150 University Avenue, Madison, Wisconsin 53706, USA \\ ${ }^{3}$ Applied Quantum Technologies, Durham, North Carolina 27707, USA
}

(Received 14 June 2010; accepted 7 September 2010; published online 28 September 2010)

\begin{abstract}
We demonstrate a scalable approach to addressing multiple atomic qubits for use in quantum information processing. Individually trapped ${ }^{87} \mathrm{Rb}$ atoms in a linear array are selectively manipulated with a single laser guided by a microelectromechanical beam steering system. Single qubit oscillations are shown on multiple sites at frequencies of $\simeq 3.5 \mathrm{MHz}$ with negligible crosstalk to neighboring sites. Switching times between the central atom and its closest neighbor were measured to be 6-7 $\mu$ s while moving between the central atom and an atom two trap sites away took 10-14 $\mu$ s. () 2010 American Institute of Physics. [doi:10.1063/1.3494526]
\end{abstract}

Scalable quantum information processing (QIP) has become a serious topic of consideration over the past decade. Among the various physical systems considered for scalable QIP implementation, individually trapped neutral atoms or ions serving as quantum bits (qubits) have emerged as leading candidates. Both of these approaches use precisely tuned lasers or microwave radiation to perform single and multiqubit gate operations ${ }^{1-4}$ and have shown the ability to trap and image multiple atomic qubits. ${ }^{5-9}$ In order to extend these quantum gates over a larger array of qubits, a scalable way of distributing laser or microwave resources is necessary. Previously, this has been accomplished using magnetic field gradients $^{10,11}$ or acousto/electro-optic deflectors. ${ }^{12,13}$ In this paper, we demonstrate a more scalable laser multiplexer utilizing microelectromechanical systems (MEMS) technology, which can easily be extended to multiple laser beams across a range of wavelengths providing the capability to address multiple trap sites simultaneously. ${ }^{14,15}$ Using a twodimensional (2D) MEMS laser steering system, Ramaninduced single qubit rotations are shown on individual ${ }^{87} \mathrm{Rb}$ atoms in a linear array with negligible impact on neighboring atoms. In addition we present a quantitative characterization of the Rabi frequency and switching times achieved in our setup.

The design of the 2D MEMS steering system, shown in Fig. 1(a), is guided by fast steering speed requirements. Beam steering is accomplished by two one-dimensional tilting micromirrors with high mechanical resonant frequencies at near critical damping. ${ }^{16}$ The micromirrors are fabricated on the same substrate, using a commercial foundry process (PolyMUMPS, offered by MEMSCAP, Inc.), ${ }^{17}$ and designed to tilt in orthogonal directions. Each micromirror consists of a gold coated polysilicon plate with a radius of $100 \mu \mathrm{m}$ that rotates about two torsional beams. The micromirror is electrostatically tilted by a potential difference between the grounded mirror plate and an applied voltage on the underlying actuation electrodes. A simple $2 f-2 f$ folded imaging system is used to optically combine the perpendicular steer-

${ }^{a)}$ Electronic mail: jungsang@duke.edu. ing axes and amplify the angular range of the system by forcing the beam to reflect off each micromirror twice, which reduces the maximum mirror tilt requirement to enable higher resonant frequencies. ${ }^{14}$ Figure $1(\mathrm{~b})$ shows the transient behavior of a mirror used in this experiment.

Individual ${ }^{87} \mathrm{Rb}$ atoms are spatially confined in far-offresonant traps (FORTs) in a similar experimental setup to the one described in Ref. 18. The FORT sites are created by a tightly focused $1064 \mathrm{~nm}$ laser beam split with a diffractive element into five spots each with a beam waist of $3.4 \mu \mathrm{m}$
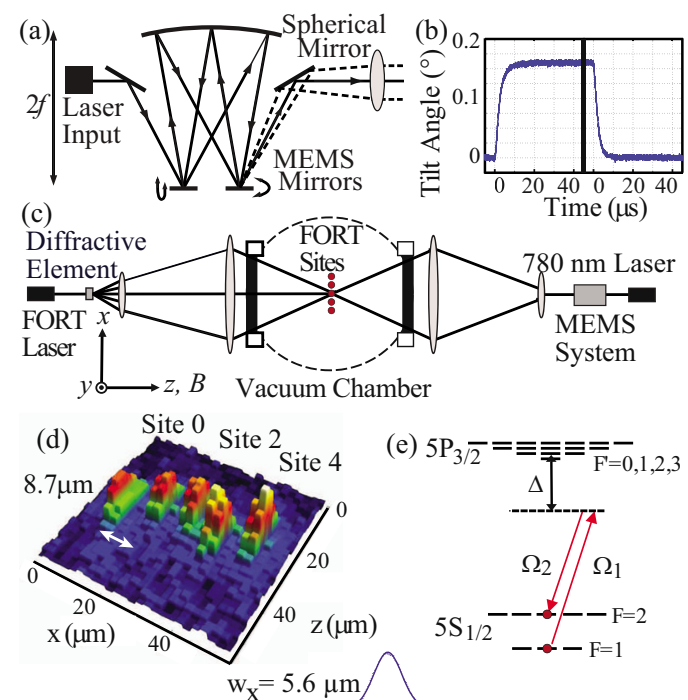

(f)

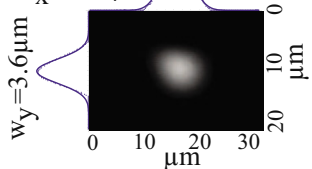

(e) $5 \mathrm{P}_{3 / 2}=\equiv \equiv \equiv \overline{\overline{\mathrm{F}}}-0$
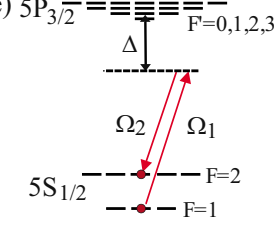

FIG. 1. (Color online) (a) MEMS steering system schematic. (b) Mirror tilt transient response when voltage is applied (left) or removed (right) at time $t=0$. (c) Schematic of the experimental setup showing the FORT beams and the $780 \mathrm{~nm}$ ground state Rabi laser. (d) Averaged fluorescence image of the five trap sites over many trap loading periods. Each site is separated by $8.7 \mu \mathrm{m}$. (e) Energy level structure showing the two photon Raman transition used for single qubit rotations. (f) Image and profile of the focused ground state Rabi laser at the atom. 
[Fig. 1(c)]. This produces a linear array of five trap sites spatially separated by $8.7 \mu \mathrm{m}$ with a peak potential depth of $\sim 4.5 \mathrm{mK}$. The FORT sites are loaded from an atom cloud confined by a magneto-optical trap (MOT). Loading is checked by collecting resonant fluorescence from the atoms on an electron-multiplying charge-coupled device camera and integrating counts over regions of interest aligned with each of the five FORT sites. The atoms are then Doppler cooled with the MOT beams to temperatures of 200-250 $\mu \mathrm{K}$. Figure 1(d) shows an averaged fluorescence image of many single atom loading periods with sufficient spacing to resolve individual atoms in each lattice site.

The qubit is defined by two magnetic field insensitive hyperfine ground states $|0\rangle \equiv\left|F=1, \quad m_{F}=0\right\rangle$ and $|1\rangle \equiv \mid F$ $\left.=2, \quad m_{F}=0\right\rangle$. Single qubit rotations between these two states are induced by a two photon Raman transition as shown in Fig. 1(e). This process uses a single ground state Rabi laser with two frequency components separated by the hyperfine splitting $\Delta \nu=6.8 \mathrm{GHz}$ generated by current modulation of a $780 \mathrm{~nm}$ diode laser. The laser is detuned from the $5 P_{3 / 2}$ excited state by $\Delta=-2 \pi \times 100 \mathrm{GHz}$ and is circularly polarized with respect to the magnetic field direction ( $z$ axis). It propagates through the MEMS steering system and focuses on the central atom (site 2) when unactuated. The beam at the atom is slightly elliptical with beam waists of $w_{x}=5.6 \mu \mathrm{m}$ and $w_{y}=3.6 \mu \mathrm{m}$ [Fig. 1(f)].

In our first experiment, initial states are prepared by applying a magnetic field to split the $m_{F}$ energy levels and optically pumping the atoms into the $|1\rangle$ state. The Rabi laser is directed to a target atom for a duration of $T$. The resulting state is then measured by selectively ejecting the atoms in the $|1\rangle$ state from the trap and detecting fluorescence from the undisturbed atoms in the $|0\rangle$ state as described in Ref. 3.

The frequency of the ground state Rabi oscillations is given by $\Omega_{R}=\Omega_{1} \Omega_{2}^{*} / 2 \Delta$ where the Rabi frequencies of the two laser side bands are $\Omega_{1}$ and $\Omega_{2}$, and the detuning from the excited state $\Delta$ is much larger than the excited state decay rate. By measuring the probability of detecting the atom in the $|0\rangle$ state as a function of the Rabi laser duration $T$, we see coherent Rabi oscillations between the two qubit states at several of the five trap sites [Fig. 2]. When the MEMS system targets site 1, the atom oscillates between the qubit states at a frequency of $3.5 \mathrm{MHz}$ and a contrast of $0.97 \pm 0.04$ [Fig. 2 (a)] while the crosstalk to neighboring atoms at sites 0 and 2 shown in (b) is negligible, within the experimental noise limit of the system. Figure 2(c) [Fig. 2(e)] shows the Rabi oscillation of the targeted site with a frequency of $3.8 \mathrm{MHz}$ $(3.1 \mathrm{MHz})$ and contrast of $1.04 \pm 0.05(0.98 \pm 0.04)$ when the MEMS system addresses site 2 (site 4) while Rabi flopping at neighboring sites are shown in Fig. 2(d) [Fig. 2(f)]. The difference in Rabi frequencies indicates a variation in the laser intensity the atom experiences at each of the trap sites. The intensity difference among sites arises from the aberrations in the MEMS folded imaging system and small actuation-induced curvature of the micromirrors but its effects can be calibrated out by adjusting the pulse duration at each site.

In addition to individual addressability, we examine the time it takes to switch the beam between atoms. First, the atom at the target site is prepared in the initial $|1\rangle$ state. Then, the MEMS system is triggered to adjust the beam path from an initial site to the target site. After a delay of $\tau$, a timed
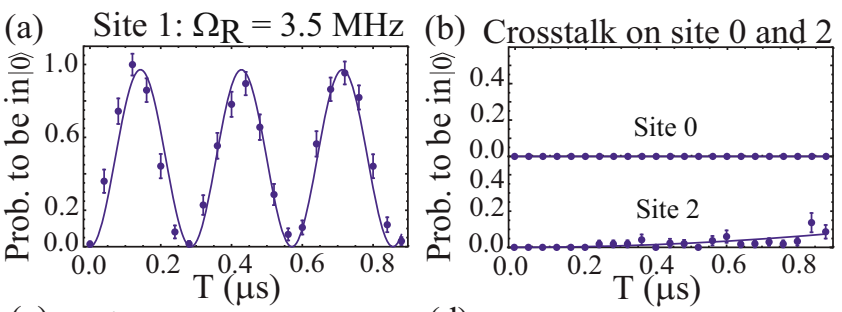

(c) Site $2: \Omega \mathrm{R}=3.8 \mathrm{MHz}$

(d) Crosstalk on site 1 and 3

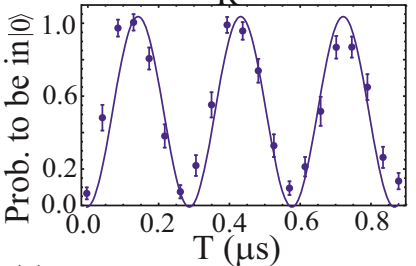

(e) Site $4: \Omega_{\mathrm{R}}=3.1 \mathrm{MHz}$
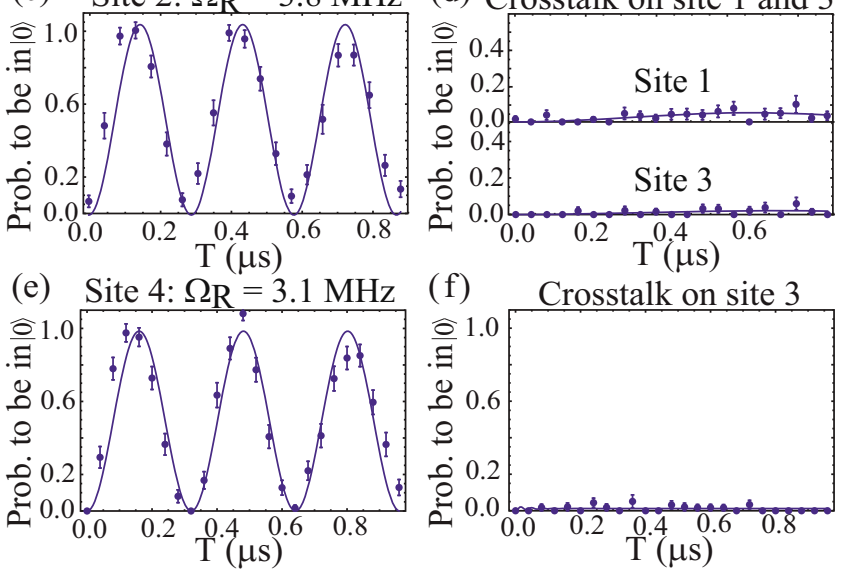

FIG. 2. (Color online) Rabi flopping on target atom and neighboring atom(s) when the laser addresses sites $[(\mathrm{a})$ and (b)] $1,[(\mathrm{c})$ and (d)] 2, and $[(\mathrm{e})$ and (f) 4 .

Rabi laser pulse flips the illuminated atom to the $|0\rangle$ state ( $\pi$-pulse), and the resulting state is measured. Since the $\pi$-pulse duration $(\sim 140 \mathrm{~ns})$ is sufficiently less than the switching time of the MEMS system $(\sim 10 \mu \mathrm{s})$, this measurement should provide an accurate indication of the fraction of the $\pi$-pulse acting on the atom for various delays $\tau$, leading to the determination of the switching time. Figure 3 shows the probability of finding the target atom in the $|0\rangle$ state as a function of $\tau$ for several configurations. When $\tau$ is small, the state of the atom does not make a complete transition to the $|0\rangle$ state as the beam has not arrived at the target location. After sufficient delay the full $\pi$-pulse impinges on the atom leading to a complete state change. The solid lines are theoretical models generated from independent transient
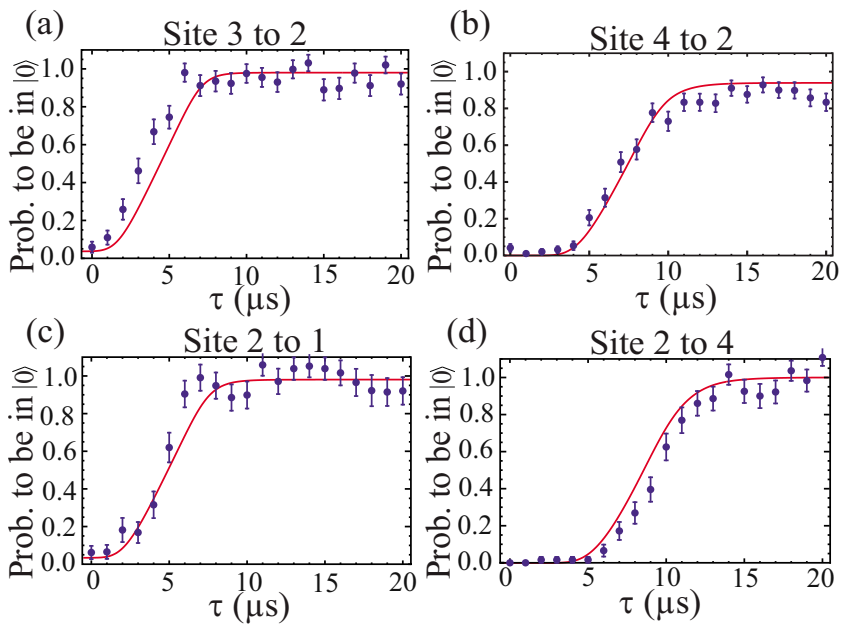

FIG. 3. (Color online) Experimental data (points) and model (solid line) showing the probability the atom has switched from the $|1\rangle$ to the $|0\rangle$ state after a delay $\tau$ between the MEMS system trigger and a $\pi$-pulse for transitions from (a) $3 \rightarrow 2$, (b) $4 \rightarrow 2$, (c) $2 \rightarrow 1$, and (d) $2 \rightarrow 4$. 
measurements of the mirror tilt and the atomic transition rate equations. The model also takes into account a uniform timing jitter distribution in $\tau$ that arises from hardware limitations in the experiment $(\sim 2 \mu \mathrm{s})$. Switching times of 6-7 $\mu$ s are measured when the MEMS system moves the laser between site 2 (center) and its neighboring sites while they increase to $10-14 \mu \mathrm{s}$ when addressing involves the outer sites. The switching is faster returning to site 2 as shown in Fig. 3 by comparing (a) and (b) with (c) and (d), respectively. This is due to well-known electrostatic softening effect in MEMS actuation, ${ }^{16}$ which results in a slower response as the MEMS system (1) addresses sites further from the center and (2) switches from the middle site to one of the outer sites compared to when it returns to the central site. We note the switching time between sites can be compensated by pretriggering if multiple independent beam paths are available in the MEMS system.

The individual qubit addressability demonstrated in this paper can be extended to arbitrary single qubit gates if the phase of the optical beams remains stable. Our experiment used copropagating Raman beams with frequency separation $\Delta \nu=6.8 \mathrm{GHz}$, where the quantum control is intrinsically insensitive to phase fluctuations due to motion on a length scale small compared to $c / \Delta \nu \sim 4.4 \mathrm{~cm}$. In the case of bichromatic beams in a counterpropagating geometry, ${ }^{3}$ phase stability at the optical wavelength scale is important for high fidelity quantum control. Further characterization of the phase stability is necessary to verify applicability of MEMS technology for such an experiment.

In summary, this paper has demonstrated individual addressability of a linear array of five trapped ${ }^{87} \mathrm{Rb}$ atoms with negligible cross talk to neighboring atoms using a scalable MEMS technology. Rabi frequencies at these sites of 3.1-3.8 $\mathrm{MHz}$ show the capability of fast single qubit gates. MEMS steering technology promises scalability to address more trap sites using more simultaneous lasers across a wide range of wavelengths at adequate switching speeds. While we have focused on its use in neutral atom QIP, it is applicable to other qubit systems in a periodic lattice including atomic ions, diamond nitrogen-vacancy color centers, and quantum dots.

This work is supported by ARO under Contract No. W911NF-08-C-0032, ARO/IARPA under Contract No. W911NF-05-1-0492, NSF Grant No. PHY-0653408, and Grant No. CCF-0546068.

${ }^{1}$ D. Leibfried, B. DeMarco, V. Meyer, D. Lucas, M. Barrett, J. Britton, W. M. Itano, B. Jelenkovic, C. Langer, T. Rosenband, and D. J. Wineland, Nature (London) 422, 412 (2003).

${ }^{2}$ F. Schmidt-Kaler, H. Haffner, M. Riebe, S. Gulde, G. P. T. Lancaster, T. Deuschle, C. Becher, C. F. Roos, J. Eschner, and R. Blatt, Nature (London) 422, 408 (2003).

${ }^{3}$ L. Isenhower, E. Urban, X. L. Zhang, A. T. Gill, T. Henage, T. A. Johnson, T. G. Walker, and M. Saffman, Phys. Rev. Lett. 104, 010503 (2010).

${ }^{4}$ T. Wilk, A. Gatan, C. Evellin, J. Wolters, Y. Miroshnychenko, P. Grangier, and A. Browaeys, Phys. Rev. Lett. 104, 010502 (2010).

${ }^{5}$ S. Seidelin, J. Chiaverini, R. Reichle, J. J. Bollinger, D. Leibfried, J. Britton, J. H. We-senberg, R. B. Blakestad, R. J. Epstein, D. B. Hume, W. M. Itano, J. D. Jost, C. Langer, R. Ozeri, N. Shiga, and D. J. Wineland, Phys. Rev. Lett. 96, 253003 (2006).

${ }^{6}$ H. C. Nägerl, W. Bechter, J. Eschner, F. Schmidt-Kaler, and R. Blatt, Appl. Phys. B: Lasers Opt. 66, 603 (1998).

${ }^{7}$ J. V. Porto, S. Rolston, B. L. Tolra, C. J. Williams, and W. D. Phillips, Philos. Trans. R. Soc. London, Ser. A 361, 1417 (2003).

${ }^{8}$ K. D. Nelson, X. Li, and D. S. Weiss, Nat. Phys. 3, 556 (2007).

${ }^{9}$ A. Lengwenus, J. Kruse, M. Volk, W. Ertmer, and G. Birkl, Appl. Phys. B: Lasers Opt. 86, 377 (2007).

${ }^{10}$ D. Schrader, I. Dotsenko, M. Khudaverdyan, Y. Miroshnychenko, A Rauschenbeutel, and D. Meschede, Phys. Rev. Lett. 93, 150501 (2004).

${ }^{11}$ S. X. Wang, J. Labaziewicz, Y. F. Ge, R. Shewmon, and I. L. Chuang, Appl. Phys. Lett. 94, 094103 (2009).

${ }^{12}$ D. D. Yavuz, P. B. Kulatunga, E. Urban, T. A. Johnson, N. Proite, T. Henage, T. G. Walker, and M. Saffman, Phys. Rev. Lett. 96, 063001 (2006).

${ }^{13}$ F. Schmidt-Kaler, H. Haffner, S. Gulde, M. Riebe, G. P. T. Lancaster, T. Deuschle, C. Becher, W. Hansel, J. Eschner, C. F. Roos, and R. Blatt, Appl. Phys. B: Lasers Opt. 77, 789 (2003).

${ }^{14}$ C. Knoernschild, C. Kim, B. Liu, F. P. Lu, and J. Kim, Opt. Lett. 33, 273 (2008).

${ }^{15}$ C. Knoernschild, C. Kim, F. P. Lu, and J. Kim, Opt. Express 17, 7233 (2009).

${ }^{16}$ C. Kim, C. Knoernschild, B. Liu, and J. Kim, IEEE J. Sel. Top. Quantum Electron. 13, 322 (2007).

${ }^{17}$ MEMSCAP, http://www.memscap.com

${ }^{18}$ E. Urban, T. A. Johnson, T. Henage, L. Isenhower, D. D. Yavuz, T. G Walker, and M. Saffman, Nat. Phys. 5, 110 (2009). 